\title{
Residual Stress Measurement in Si-based Multilayer Structure by Micro-Raman Spectroscopy
}

\author{
Wei Qiu ${ }^{1,2,{ }^{*}, \text { CuiLi Cheng }}{ }^{1}$, YuCheng Zhao ${ }^{1}$, Qiu Li ${ }^{3}$ \\ ${ }^{1}$ Tianjin Key Laboratory of Modern Engineering Mechanics, Department of Mechanics, Tianjin University, Tianjin, China \\ ${ }^{2}$ LANE, Department of Electrical Engineering, University of Nebraska-Lincoln, NE, USA \\ ${ }^{3}$ Tianjin Key Laboratory of High Speed Cutting and Precision Machining, Tianjin University of Technology and Education, Tianjin, China
}

Email address:

qiuwei@tju.edu.cn (Wei Qiu)

\section{To cite this article:}

Wei Qiu, CuiLi Cheng, YuCheng Zhao, Qiu Li. Residual Stress Measurement in Si-based Multilayer Structure by Micro-Raman Spectroscopy. Optics. Special Issue: Optical Techniques for Deformation, Structure and Shape Evaluation. Vol. 4, No. 3-1, 2015 , pp. 33-38. doi: 10.11648/j.optics.s.2015040301.18

\begin{abstract}
Si-based multilayer structures, such as porous silicon (PS) and germanium silicon ( $\varepsilon$-Si), are widely used in current microelectronics and Micro-electromechanical Systems (MEMS). During the preparing process of Si-based multilayer structures, some inhomogeneous residual stress is induced, maybe finally leading to structure failure. Micro-Raman Spectroscopy (MRS) is regarded as an effective method for intrinsic stress measurement. In this work, MRS is applied to analysis the residual stress distribution along the cross section of Si-based multilayer structure. Raman experimental results show that there are noticeable residual stress in both silicon substrate and the films (including porous silicon film and germanium-silicon buffer layers). The residual stress is linearly varied in most regions, which leads to an overall warp of the structure. While nonlinear variation of residual stress appears at the interface between different films and Si-substrate. Based on the experimental results, a spectra-mechanical model for analyzing the transversely-isotropic material like porous silicon was presented. A set of detailed Raman stress relationship of porous silicon was achieved. For the study of strained silicon wafer, the structural analyses by using high-resolution transmission electron microscope (HR-TEM) were cooperated with the residual stress measurement by Micro-Raman. And an analysis procedure of the residual stress evaluation on multilayer germanium silicon structures was introduced.
\end{abstract}

Keywords: Residual Stress, Si-based Multilayer Structure, Micro-Raman Spectroscopy, Porous Silicon, Germanium Silicon

\section{Introduction}

Si-based multilayer structures are, and will still be in the imaginable future, the majority of the microelectronics and micro-electromechanical Systems (MEMS). For instance, because of its special properties including large specific surface area, high reactivity, diversiform topological structure and photo/electro-luminescence, porous silicon has been widely used in recent years in MEMS, optoelectronic/microelectronic devices and clinical medicine/pathology bio-sensors [1,2]. Meanwhile, strained silicon materials with germanium silicon buffer layers can improve the hole (or electron) mobilities of the NMOS (or PMOS) transistors, hence they have become standard units in current CPU and GPU chips in computers and mobile communication devices [3].

Nevertheless, intrinsic and/or processing residual stress always introduced into these Si-based structures during manufacturing [4]. Thus, it is necessary to study the experimental method of the effective and non-destructive residual stress measurement for Si-based structures or devices.

Several methods have been used to characterize residual stresses in thin films such as curvature method [5], X-ray diffraction [6] and MRS [7]. Basically, curvature method detects the change of the substrate curvature before and after film deposition and calculates the stress of the film by using the Stoney equation [8]. This method provides an average stress value over the film area. Owing to its approximate algorithms of stress distribution, it is not suitable for a thin film with asymmetric stress distribution. X-ray diffraction measures stress by detecting the lattice deformation, and it is applicable to study the residual stresses in different layers in different depths of multi-layer structures. However, it is hardly applicable for the mechanical measurement on 
microelectronic devices due to its inherent limitations in spatial solution and accuracy.

In recent years, the residual stress measurement method based on MRS has a remarkable development for its special advantages such as non-destructive, noncontact, high spatial resolution $(1 \mu \mathrm{m})$ [9-12]. The mechanical application of MRS requires that the quantitative relationship between Raman shift and stress of the measured material is determined. Some common crystal materials, such as single-crystal silicon, polysilicon, $\mathrm{GaN}$ and $\mathrm{SiC}$, have been well studied [13-15]. While quite a few materials, even including some widelyused Si-based materials, are still requires basic investigation on Raman-mechanical methods [16,17]. For instance, some Si-based materials, such as porous silicon, have the different strain-stress relationship with that of the crystal silicon. Some Si-based alloy materials, such as germanium silicon, have complex Raman effects whose Raman shift is not only induced by strain. To measure the residual stress inside these materials, the Raman shift to stress relationship should be investigated firstly and quantificationally.

This paper presents two methodologic works on the residual stress measurements of Si-based multilayer structures including porous silicon and germanium silicon.

\section{Residual Stress Analysis on Porous Silicon}

\subsection{Samples and Experiments}

The PS samples were fabricated by electrochemical etching on 2" (100) silicon wafers ( $\mathrm{p}^{+}$-type, 0.01 0.02 $\Omega \cdot \mathrm{cm}$ ) with a 1:1 HF/ethanol solution. The PS layer was about $30 \mu \mathrm{m}$ in thickness and its porosity was estimated as $60 \%$ by means of usual gravimetric method.

The experiments on porous silicon samples applied a Ranishaw RM2000 microscopic confocal Raman system with a 514nm Ar+ laser exciting light whose output was regulated to $1 \%(0.23 \mathrm{mw})$ to avoid the laser-heating effect. A $50 \times$ Olympus objective was selected; hence the spot size of incident laser was about $2 \mu \mathrm{m}$ in diameter.

\subsection{Raman-Mechanical Model of Porous Silicon}

The spectrum of Raman scattering is composed of a few characteristic peaks corresponding to all the Raman-visible phonon modes. The location of each Raman characteristic peak is mostly determined by the vibration energy of its phonon mode, but it may vary more or less when the crystal lattice of the material deforms leading to an energy variation of lattice vibration. Take the monocrystalline silicon (abbr. C$\mathrm{Si}$ ) as an example. The Raman spectrum of strain-free C-Si exhibits a single peak at about $520 \mathrm{~cm}^{-1}$. This peak corresponds to the triply degenerate optical phonons, and its location may shift towards lower/higher frequencies when the lattice in tensile/compression, respectively. Since the macro-deformation of crystal (namely strain) can be regarded as the statistical accumulation of the micro-deformation of lattice, strain and stress measurement can be realized by detecting the Raman shift variation of the samples to be measured and applying the generalized Hooke's law.

The lattice dynamics secular equation of diamond-type crystal is as (1) [13].



$$
\begin{aligned}
& 2 r \varepsilon_{x z} \quad 2 r \varepsilon_{y z} \quad\left[\begin{array}{l}
q\left(\varepsilon_{x x}+\varepsilon_{y y}\right) \\
+p \varepsilon_{z z}-\lambda
\end{array}\right]
\end{aligned}
$$

where $p, q$ and $r$ are material constants named as phonon deformation potentials, $\varepsilon_{i j}(i, j=x, y, z)$ are components of the strain tensor, and $\lambda=w^{2}-w_{0}^{2}, w$ and $w_{0}$ are the Raman shift of strained and strain-free sample, respectively. Besides, the Raman shift variation $\Delta w$ induced by strain is smaller in magnitude than $w_{0}$,

$$
\Delta w=w-w_{0} \approx \frac{w^{2}-w_{0}^{2}}{2 w_{0}}=\frac{\lambda}{2 w_{0}}
$$

Therefore, the relation between $\Delta w$ and $\varepsilon_{i j}$ is built up by solving Secular (1) for the eigenvalue $\lambda$.

As for the porous silicon in this work, a photo (Fig. 1a) of the cross-section sample obtained using a field-emission scanning electron microscope (FE-SEM) shows that the topological structure roughly resembles a bushy flourishing forest as Fig. $1 \mathrm{~b}$ illustrates. The pillars are nearly parallel with each other and vertical to the plane of PS film. The diameters of both pores and pillars are several tens of nanometers, about one magnitude larger than the size of grains and subpores. All these measurements show that the porous silicon belongs to the transversely-isotropic material with diamondtype crystal structure.

By substituting the strain-stress relationship of the transversely-isotropic material into (1) and applying (2) at same time, the general Raman-mechanical relationship of transversely-isotropic material with diamond-type crystal can be achieved. While it should be noticed that there exists no normal stress on the measurement surface of material, i.e. $\sigma_{z z}$, $\sigma_{x x}$ or $\sigma_{y y}$ equals to zero on $x-y, y-z$ or $x-z$ surface, respectively And the out-plane stress component $\sigma_{z z}$ is usually negligible as for the film structure (shown in Fig.1(c)) without external loading. Hence the Raman shift to stress relationship for the residual stress measurement of the transversely-isotropic material is as follows.

$$
\left\{\begin{array} { l l } 
{ \Delta w _ { 1 } = \Psi _ { 1 1 } \sigma _ { y y } } & { ( y - z ) } \\
{ \Delta w _ { 2 } = \Psi _ { 1 1 } \sigma _ { x x } } & { ( x - z ) , } \\
{ \Delta w _ { 3 } = \Psi _ { 3 3 } ( \sigma _ { x x } + \sigma _ { y y } ) } & { ( x - y ) }
\end{array} \left\{\begin{array}{l}
\Psi_{11}=\frac{-p v_{x y}+q\left(1-v_{x z}\right)}{2 w_{0} E_{x}} \\
\Psi_{33}=\frac{-p v_{x z}+q\left(1-v_{x y}\right)}{2 w_{0} E_{x}}
\end{array}\right.\right.
$$

where $E_{x}$ are elastic modulus in $x$ axis, $v_{x y}$ and $v_{x z}$ Poisson's 
ratios in $x-y$ and $x-z$ surface, respectively.
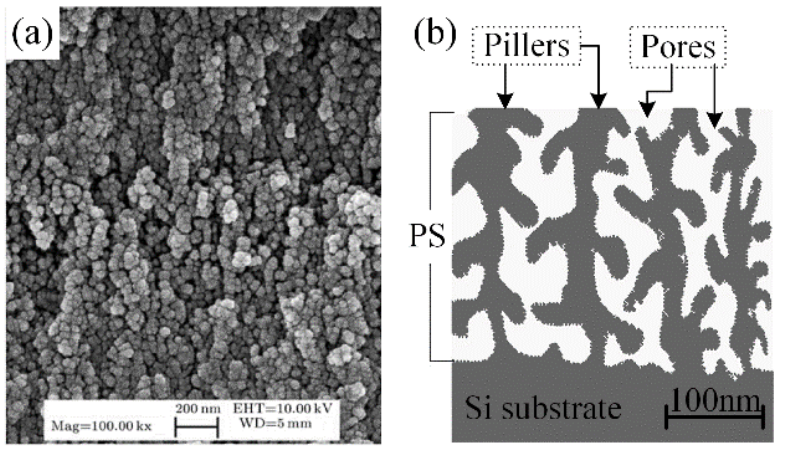

(c) $\mathrm{z}[001]$

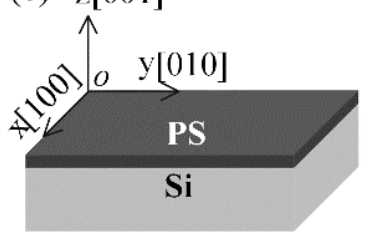

(d)

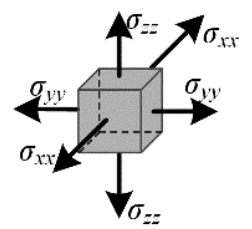

Figure 1. (a) field-emission scanning electron microscopy (FE-SEM) photograph of PS. (b) Schematic of PS topological structure. (c) Schematic of macrostructure and coordinate system of PS (d) the stress state of main unit body.

It can be seen from (3) that some physical and mechanical parameters of porous silicon material are required to determine the coefficients $\Psi_{11}, \Psi_{13}$ and $\Psi_{33}$. These parameters includes $w_{0}, p$ and $q, E_{x}, E_{z}, v_{x y}$ and $v_{x z}$. For the porous silicon with $60 \%$ porosity, $p, q$ and $w_{0}$ are regarded as approximate to those of single-crystal silicon since the crystal grains in porous silicon are dominated by single-crystal with a larger lattice size $[18,19]$. Thus, $w_{0}=520 \mathrm{~cm}^{-1}, p=-1.85 w_{0}{ }^{2}$ and $q=$ $-2.31 w_{0}^{2}$ [20]. Besides, $v_{x z}=0.09$ [21]. The elastic modulus in $x$ and $z$ of porous silicon samples were detected by using a MTS Nanoindenter XP with by a pyramidal Berkovich diamond indenter, achieving $E_{x}=23.987 \mathrm{GPa}$. The Poisson's ratio $v_{x y}$ were measured by applying the digital speckle correlation method (DSCM) during step-by-step uniaxial compressive loading on the PS sample. The result is $v_{x y}=$ 0.19 . Hence the he Raman shift to stress relationship for the residual stress measurement of the porous silicon with $60 \%$ porosity is as follows. And those with other porosity can also be achieved by the same method,

$$
\begin{cases}\sigma_{y y}=-52.7 \Delta w_{1} & (y-z) \\ \sigma_{x x}=-52.7 \Delta w_{2} & (x-z) \\ \sigma_{x x}+\sigma_{y y}=-54.1 \Delta w_{3} & (x-y)\end{cases}
$$

\subsection{Results and Discussion}

Fig. 2(a) shows the variation of Raman shift along the thickness direction of sample obtained by experiments, where the $w_{0}=519.08 \mathrm{~cm}^{-1}$ was provided from the Raman detections on the stress-free wafer similar to those prepared into porous silicon sample. The distribution of residual stress on PS film layer and the substrate layer of single-crystal silicon along the thickness direction is shown in Fig. 2(b) by using the referrence shift $519.08 \mathrm{~cm}^{-1}$ and the Raman shift to stress coefficient as $-52.7 \mathrm{Mpa} / \mathrm{cm}^{-1}$ and $435 \mathrm{Mpa} / \mathrm{cm}^{-1}$ for PS and silicon substrate, respectively.
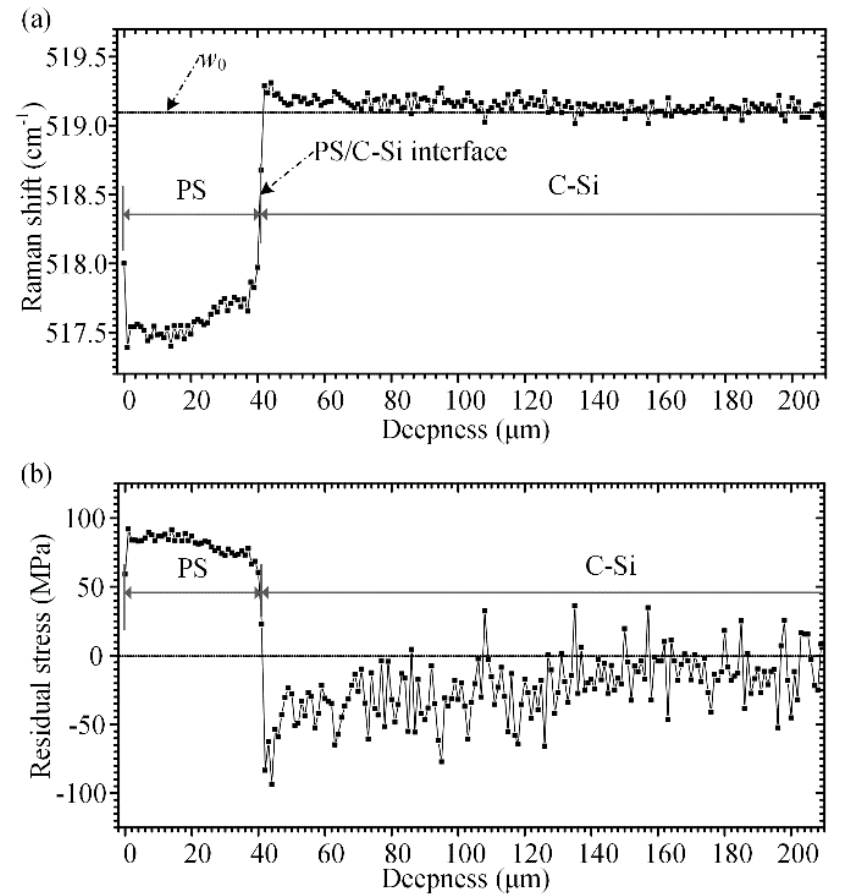

Figure 2. The distribution of (a) Raman shift and (b) residual stress in the PS-film structure along the thickness direction(the red line indicates the change trend of the residual stress).

Fig. 2(b) shows that the residual stress in porous silicon material is tensile stress which is released somewhat in a small area near the surface. The stress inside PS is relatively stable with the value about $85 \mathrm{Mpa}$, which has the same magnitude with the results obtained by using substrate curvature method [5], and this tensile stress is the primary cause of PS film fracture. The stress near the interface (or rather interphase) area between porous silicon film and silicon substrate has a large variation gradient. It changes sharply from tensile stress to maximum compressive stress. This is because numerous lattice mismatch was introduced in this area during the fabrication process of porous silicon. Except for the interface area, the compressive stress inside silicon substrate decreased gradually with almost linearity, and it can be deduced that the stress in the silicon substrate increased linearly to tensile stress from the stress variation trend given by test. It is reasonable because the tensile and the compressive stress of the cross section should be balance naturally. Therefore, the residual stress generated in PS film during the fabrication may cause the warpage of the whole structure.

\section{Residual Stress Analysis on $\varepsilon-\mathrm{Si} / \mathbf{G e}-\mathrm{Si}$}

\subsection{Samples and Experiments}

The $\varepsilon$-Si/Ge-Si material in this work was prepared through ultra-high vacuum chemical vapour epitaxy (UHVCVD) [22]. 
$\mathrm{SiH}_{2} \mathrm{Cl}_{2}$ gas was used as the $\mathrm{Si}$ source, $\mathrm{GeCl}_{4}$ as a $\mathrm{Ge}$ source gas, and $\mathrm{H}_{2}$ as the carrier gas. As the substrate, 5" (100) monocrystalline silicon wafer with $630 \mu \mathrm{m}$ in thickness was pre-treated by steeped in $\mathrm{H}_{2} \mathrm{SO}_{4}: \mathrm{H}_{2} \mathrm{O}_{2}=3: 1$ solution for 10 minutes to remove the organic soil on the surfaces and then rinsing for $45 \mathrm{~s}-50 \mathrm{~s}$ in $10 \% \mathrm{HF}$ solution to remove the oxide layer. The growth temperature was $900^{\circ} \mathrm{C}$, and the pressure was $1.33 \times 10^{4} \mathrm{~Pa}$. The component of germanium was increased linearly, forming a graded silicon germanium $\left(\mathrm{Ge}_{\mathrm{x}} \mathrm{Si}_{1-\mathrm{x}}, \mathrm{x}=0 \rightarrow 0.2\right)$ buffer layer of $3 \mu \mathrm{m}$ thickness. Then, the growth rate was low down and the component of germanium was hold on to obtain a relaxed silicon germanium $\left(\mathrm{Ge}_{0.2} \mathrm{Si}_{0.8}\right)$ buffer layer of $0.8 \mu \mathrm{m}$ thickness. Finally, a strain silicon cap layer of $10 \mathrm{~nm}$ was grown at $750^{\circ} \mathrm{C}$ and $1.33 \times 10^{4} \mathrm{~Pa}$.

The cross-section sample was prepared as follows. The Sibased $\varepsilon$-Si/Ge-Si mutilayer wafer was cut to numbers of pieces. Two of them were pasted with $\varepsilon$-Si surfaces face-toface bonded by using epoxy resin, and two C-Si pieces, cut from $2 "(100)$ monocrystalline silicon wafer with $430 \mu \mathrm{m}$ in thickness, were pasted outside the two $\varepsilon$-Si ones, respectively. The piece group was cut to thin strips, and then the crosssection surfaces were polished to roughness $R_{\mathrm{a}}<0.2 \mathrm{~nm}$.

The Raman experiments on the cross-section sample applied a Ranishaw InVia microscopic confocal Raman system with a $532 \mathrm{~nm}$ laser exciting light whose output was regulated to $10 \%$. A $50 \times$ Leica objective was selected; hence the spot size of incident laser was about $1 \mu \mathrm{m}$ in diameter.

After the Raman experiments, the cross-section sample was further prepared for the measurements by using transmission electron microscopy.

\subsection{Raman-Mechanical Model of Ge-Si}

The Raman spectrum of germanium silicon, shown in Fig 3 , has three mean peaks: Si-Si band around $500 \mathrm{~cm}^{-1}, \mathrm{Ge}-\mathrm{Si}$ band around $400 \mathrm{~cm}^{-1}$ and $\mathrm{Ge}-\mathrm{Ge}$ band around $300 \mathrm{~cm}^{-1}$. When Si element dominate the Ge-Si alloy, such as the buffer material in this work, the $\mathrm{Si}-\mathrm{Si}$ band is enough strong and narrow to be applied for mechanical measurement.

The basic Raman-mechanical model for crystal silicon (C$\mathrm{Si}$ ) follows the lattice dynamics secular equation as (1). As for the backscattering measurement of the sample surface, the third eigenvalue equation is Raman visible [13].

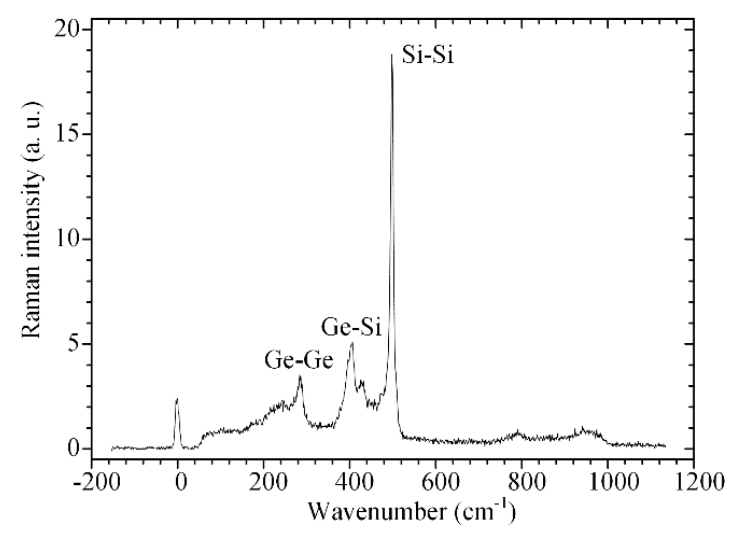

Figure 3. Raman spectrum of germanium silicon.

$$
\Delta w_{3}=\frac{p \varepsilon_{33}+q\left(\varepsilon_{11}+\varepsilon_{22}\right)}{2 w_{o}}
$$

where $w_{0}=520 \mathrm{~cm}^{-1}, p=-1.85 w_{0}^{2}, q=-2.31 w_{0}^{2}$, and Poisson's Ratio $\mu=0.278$. Hence (5) becomes (6) in the biaxial stress state.

$$
\varepsilon_{11}=\varepsilon_{22}=-1.209 \times 10^{-3} \Delta w_{3}
$$

While, duo to the influence of the phonon confinement, the wavenumber change of Si-Si band in the Raman spectrum of germanium silicon is not only induced by strain. T.S. Perova [23] presented an empirical relationships of wavenumber $w_{\mathrm{Si}-}$ Si, Ge content $x$ and strain $\varepsilon$.

$$
w_{\mathrm{Si}-\mathrm{Si}}=520-68 x-830 \varepsilon
$$

Equation (7) can be transformed into (8)

$$
\varepsilon=-1.205 \times 10^{-3}\left(\Delta w_{\mathrm{Si}-\mathrm{Si}}+68 x\right)
$$

As compared with (6), it can be seen that the Raman shift factor of the germanium silicon are basically similar to that of the monocrystalline silicon. The only difference is the $68 \mathrm{x}$ compensation of the wavenumber change for the influence of the phonon confinement. Therefore, the Raman-strain relationship of the germanium silicon in the biaxial stress state can be regarded as (9).

$$
\varepsilon=-1.209 \times 10^{-3}\left(\Delta w_{3}+68 x\right)
$$

As for the backscattering measurement of cross-section sample, the second eigenvalue equation is Raman visible [13]. Meanwhile, the normal stress of cross-section surface is totally released and the material is in uniaxial stress state (the stress parallels the interface direction). Hence the Ramanstrain relationship for $\mathrm{C}-\mathrm{Si}$ is (10).

$$
\Delta w_{2}=\frac{-p \mu+q(1-\mu)}{2 w_{\mathrm{o}}} \varepsilon_{11} \Rightarrow \varepsilon_{11}=-3.334 \times 10^{-3} \Delta w_{2}
$$

And that for germanium silicon is (11).

$$
\varepsilon=-3.334 \times 10^{-3}\left(\Delta w_{2}+68 x\right)
$$

\subsection{Results and Discussion}

Fig. 4a shows the structure of the cross-section sample near the multilayer interface region. Fig.4b gives the SEM image of this region. Fig. 4c shows a high resolution TEM image achieved near the $\varepsilon$-Si layer and Fig. $4 \mathrm{~d}$ is a low resolution TEM image near the germanium silicon buffer layer. It can be seen from Fig. $4 b \sim d$ that the strained silicon layers, the graded and relaxed buffer layers all have their designed thicknesses, via $\sim 10 \mathrm{~nm}, 3 \mu \mathrm{m}$ and $0.8 \mu \mathrm{m}$. Besides, there are plenty of dislocations concentrating in the $\mathrm{Ge}_{\mathrm{x}} \mathrm{Si}_{1-\mathrm{x}}$ layer. While, the $\mathrm{Ge}_{0.2} \mathrm{Si}_{0.8}$ layer and the $\mathrm{Si}$ substrate of the sample are clean and flat, without visible defects/dislocations. 




(a)

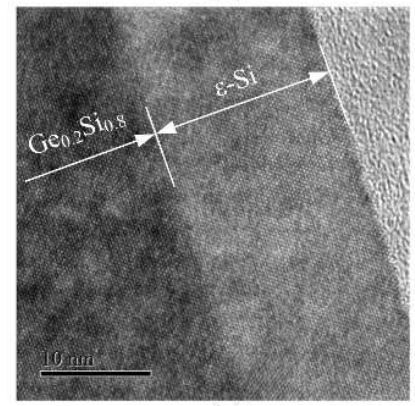

(c)

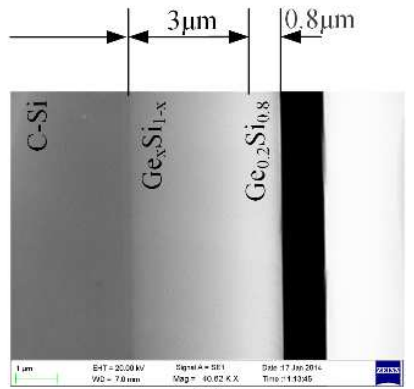

(b)

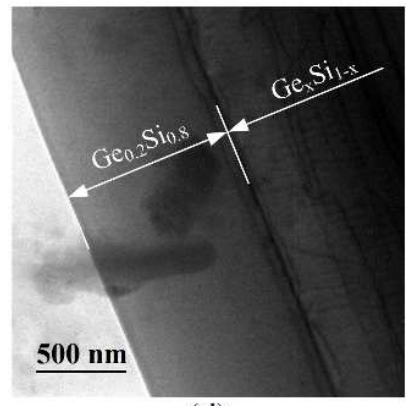

(d)
Figure 4. (a) schematic of the multilayer interface region on the crosssection sample, (b) SEM image of the region as (a), (c) high resolution TEM image near the $\varepsilon-S i$ layer, (d) low resolution TEM image near the Ge-Si buffer layer.

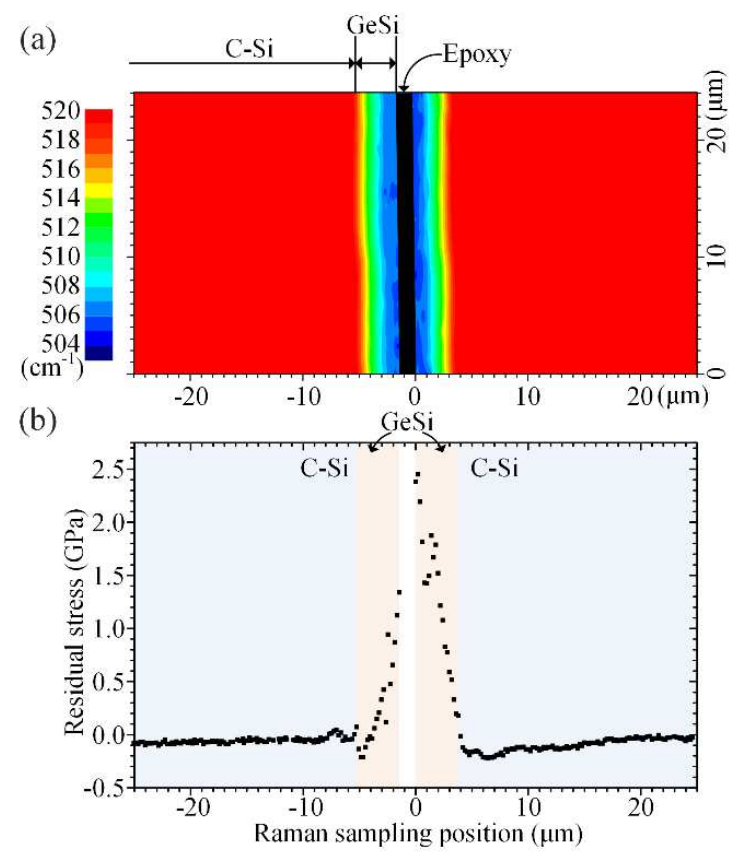

Figure 5. (a) Peak location image obtain by Raman mapping on the crosssection sample, (b) Residual stress distribution along the deepness direction.

Fig. 5a shows the image of peak location obtain by Raman mapping on the cross-section sample at a $50 \times 24 \mu \mathrm{m}^{2}$ region near the multilayer interfaces. The step lengths of Raman mapping along the parallel and vertical direction to the interfaces are $1.2 \mu \mathrm{m}$ and $0.2 \mu \mathrm{m}$, respectively. From both the Fig.5a, it can be seen that the Raman shift changes sightly inside the C-Si substrate but acutely inside the buffer layers.
The residual stress of each position inside the C-Si substrate can be achieved directly by using (10) and regarding the Young's Module of C-Si as $130 \mathrm{GPa}$, where $w_{0}$ $=519.78 \mathrm{~cm}^{-1}$ was calibrated on a silicon wafer of the same production batch with $\varepsilon$-Si sample in this work and at the same day of above measurements. The residual stress inside the germanium silicon buffer layers were achieved by using (11) and regarding the Young's Module of Ge-Si as following the ROM rule [24]. Hence the residual stress distribution along the deepness direction of this multi-layer structure is given as Fig. 5b. It can be seen there exists serious residual stress inside the buffer layers.

\section{Conclusions}

In summary, the Raman-mechanical study is based on the basic understanding on the wavenumber-strain-stress relationship. There exist a need methodologic work for some materials whose either former part of the relationship (viz. wavenumber to strain, such as the germanium silicon) or latter part (viz. strain to stress, such as porous silicon) are different with that of the traditional materials.

In this work, a spectra-mechanical model suitable for transversely-isotropic material like porous silicon was presented. A mechanical method of the residual stress evaluation by amending the strain-induced Raman shift for the germanium silicon was investigated. Based on above, the distributions of residual stress along the deepness inside the Si-based multilayer structures were measured by using micro-Raman spectroscopy.

\section{Acknowledgments}

This work is financially supported by the National Basic Research Program of China (No.2012CB937500), the National Natural Science Foundation of China (grant Nos. 11227202, 11372217 and 11272232), the Program for New Century Excellent Talents in University (Grant no. NCET-13) and China scholarship Council (201308120092).

\section{References}

[1] V. S. Y. Lin, K. Motesharei, K. P. S. Dancil, M. J. Sailor and M. R. Ghadiri, "A porous silicon-based optical interferometric biosensor," Science, vol. 278, pp. 840-843, 1997

[2] P. Steiner, and W. Lang, "Micromachining applications of porous silicon," Thin Solid Films, vol. 255, pp. 52-58, 1995.

[3] N. Hayazawa, M. Motohashi, Y. Saito, H. Ishitobi, A. Ono, T. Ichimura, P. Verma and S. Kawata, "Visualization of localized strain of a crystalline thin layer at the nanoscale by tipenhanced Raman spectroscopy and microscopy," J. Raman Spectro. vol. 38, pp. 684-696, 2007.

[4] D. Papadimitriou, J. Bitsakis, J. M. López-Villegas, J. Samitier and J. R. Morante, "Depth dependence of stress and porosity in porous silicon: a micro-Raman study," Thin Solid Films, vol. 349, pp. 293-297, 1999. 
[5] G. C. A. M. Janssen, M. M. Abdalla, F. Van Keulen, B. R. Pujada and B. Van Venrooy, "Celebrating the 100th anniversary of the Stoney equation for film stress: Developments from polycrystalline steel strips to single crystal silicon wafers," Thin Solid Films. vol. 517, pp. 1858$1867,2009$.

[6] S. Doyle, V. A. Chernenko, S. Besseghini, A. Gambardella, M. Kohl, P. Müllner and M. Ohtsuka, "Residual stress in Ni-MnGa thin films deposited on different substrates," The European Physical Journal-Special Topics, vol. 158, pp. 99-105, 2008.

[7] X. Pan, C. W. Tan, J. Miao, J. Kasim, Z. Shen and E. Xie, "The stress analysis of Si MEMS devices by micro-Raman technique," Thin Solid Films, vol. 517, pp. 4905-4908, 2009.

[8] G. G. Stoney, "The tension of metallic films deposited by electrolysis", Proc. Roy. Soc. London. Series A, vol. 82, pp. 172-175, 1909.

[9] Q. Li, W. Qiu, H. Y. Tan, J. G. Guo and Y. L. Kang, "MicroRaman spectroscopy stress measurement method for porous silicon film," Optics and Lasers in Engineering, vol. 48, pp.1119-1125, 2010.

[10] W. Qiu, Y. L. Kang, Z. K. Lei, Q. H. Qin, Q. Li and Q. Wang, "Experimental study of the Raman strain rosette based on the carbon nanotube strain sensor," J. Raman Spectro., vol. 41, pp. 1216-1220, 2010.

[11] W. Qiu, Q. Li, Z. K. Lei, Q. H. Qin, W. L. Deng and Y. L. Kang, "The use of a carbon nanotube sensor for measuring strain by micro-Raman spectroscopy," Carbon, vol. 53, pp. 161-168, 2013.

[12] W. Qiu and Y. L. Kang, "Mechanical behaviour study of microdevice and nanomaterials by Raman spectroscopy," Chinese Science Bulletin, vol. 59, pp. 2811-2824, 2014.

[13] I. D. Wolf, "Stress measurements in Si microelectronics devices using Raman spectroscopy," J. Raman Spectro. vol. 30, pp. 877-883, 1999.

[14] E. Anastassakis and E. Liarokapis, "Polycrystalline Si under strain: Elastic and lattice-dynamical considerations," J. Appl. phys., vol. 62, pp. 3346-3352, 1987.
[15] F. Demangeot, J. Frandon, M. A. Renucci, O. Briot, B. Gil and R. L. Aulombard, "Raman determination of phonon deformation potentials in $\alpha-\mathrm{GaN}$," Solid state communications, vol. 100, pp. 207-210, 1996.

[16] Y. Kang, Y. Qiu, Z. Lei and M. Hu, “An application of Raman spectroscopy on the measurement of residual stress in porous silicon," Optics and lasers in engineering, vol. 43, pp. 847-855, 2005.

[17] W. Qiu, Y. L. Kang, Q. Li, Z. K. Lei and Q. H. Qin, "Experimental analysis for the effect of dynamic capillarity on stress transformation in porous silicon,” Appl. Phys. Lett., vol. 92, 041906, 2008.

[18] O. Bisi, S. Ossicini and L. Pavesi, "Porous silicon: a quantum sponge structure for silicon based optoelectronics," Surface science reports, vol. 38, pp. 1-126, 2000.

[19] S. Manotas, F. Agulló-Rueda, J. D. Moreno, F. Ben-Hander and J. M. Martınez-Duart, "Lattice-mismatch induced-stress in porous silicon films," Thin Solid Films, vol. 401, pp. 306-309, 2001.

[20] E. Anastassakis, A. Cantarero and M. Cardona, "Piezo-Raman measurements and anharmonic parameters in silicon and diamond," Phys. Rev. B, vol. 41, 7529, 1990.

[21] K. Barla, R. Herino, G. Bomchil, J. C. Pfister and A. Freund, "Determination of lattice parameter and elastic properties of porous silicon by X-ray diffraction," Journal of Crystal Growth, vol. 68, pp. 727-732, 1984.

[22] R.R. Liang, J. Wang, J. Xu, "Fabrication of High Quality SiGe Virtual Substrates by Combining Misfit Strain and Point Defect Techniques," Tsinghua Science and Technology, vol. 14(1), pp. 62-67, 2009.

[23] T. S. Perova, K. Lyutovich, E. Kasper, A. Waldron, M. Oehme and R. A. Moore, "Stress determination in strained-Si grown on ultra-thin SiGe virtual substrates," Materials Science and Engineering B, vol. 135, pp. 192-194, 2006.

[24] S. P. Baker and E. Arzt, "Elastic stillness constants of SiGe," Properties of strained and relaxed Silicon Germanium, E. Kasper Eds. London: INSPEC, 2015, pp. 67-69. 